\title{
Thermal characterization of novel aliphatic polyesters with ether and thioether linkages
}

\author{
M. Soccio, N. Lotti, L. Finelli. A. Munari \\ "Dipartimento di Chimica Applicata e Scienza dei Materiali, Università di Bologna, Via \\ Terracini 28, 40131 Bologna, Italy; fax ++390512093222; e-mail: \\ nadia.lotti@mail.ing.unibo.it
}

(Received: 07 April, 2009; published: 25 August, 2010)

\begin{abstract}
Several novel ether or thioether linkage containing aliphatic polyesters and poly(alkylene dicarboxylate)s were synthesized for comparison and characterized in terms of chemical structure and molecular weight. The thermal behavior was examined by thermogravimetric analysis and differential scanning calorimetry. All the polymers showed a good thermal stability, even though lower for the ether or thioether linkage-containing polyesters. The decrement of the thermal stability appears to be more relevant in the case of the presence of sulphur atoms. At room temperature the samples appeared semicrystalline, except PTTDG and PDEDG, which were viscous oils; the effect of the introduction of ether or thioether group was an increment of the $T_{g}$ value, a decrement of the melting temperature and a significant decrease of the crystallization rate. The entity of the variations was found to be affected by the kind of group introduced, and the trend observed can be explained on the basis of atom electronegativity and dimensions.
\end{abstract}

\section{Introduction}

Biodegradable polymers have been extensively used in biomedical applications, for example as biomaterials for constructing absorbable bone plates, other surgical fixation devices, surgical sutures, and controlled release drug carriers [1-3]. The biocompatibility, toxicity, and immunogenicity of polymers play important role in these applications; other relevant factors concern a predictable rate of biodegradation and deliverable mechanical properties. In this view, aliphatic polyesters have been attracting considerable attention as in many cases they combine the features of biodegradability and biocompatibility with physical and chemical properties comparable with those of some of the most extensively used polymers, like LDPE, PP, etc. Moreover, they can be processed into various forms, such as fibers, films and injection-moulded devices [1-3].

There is also a great current interest on polyesters containing ether-groups: the presence of ether linkages in the chain seems indeed to increase the hydrophilic character of the polymer, favouring its dissolution under environmental conditions and/or enhancing the affinity with living cells $[4,5]$. This latter aspect is very important because it can be correlated with the capacity of some polymeric biomaterials to favour the regeneration of tissues through their interaction with the cells. Hence, surgical implants made from such biomaterials could be used as temporary scaffolds in tissue engineering. Basic research on the relationship between structure and properties permits to design and synthesize a great variety of biodegradable polymers to fulfil the demands in practical applications. 
In this view, it appeared interesting to prepare novel biodegradable ether-group containing polyesters, the corresponding poly(thioether esters) and polyesters, these for comparison. All the synthesized polymers were characterized in terms of molecular and thermal properties, in order to investigate the effect of the introduction of ethero atoms ( $\mathrm{S}$ or $\mathrm{O}$ ) in the chain of an aliphatic polymer.

\section{Results and discussion}

The chemical structures of the polyesters synthesized are reported in the following:

poly (trimethylene glutarate) (PTG)<smiles></smiles>

poly(pentamethylene pimelate) (PPP)<smiles>COCCCCCOC(=O)CCCCCC(C)=O</smiles>

poly(trimethylene diglycolate) (PTDG)<smiles>COCCCOC(=O)COCC(C)=O</smiles>

poly(tetramethylene diglycolate) (PTTDG)<smiles>COCCCCOC(=O)COCC(C)=O</smiles>

poly(diethylene diglycolate) (PDEDG)<smiles>COCCOCCOC(=O)COCC(C)=O</smiles>

poly(trimethylene thiodiglycolate) (PTTDG)<smiles>COCCCOC(=O)CSCC(C)=O</smiles>

poly(tetramethylene thiodiglycolate) (PTTTDG)<smiles>COCCCCOC(=O)CSCC(C)=O</smiles>

poly(thiodiethylene thiodiglycolate) (PTDETDP)<smiles>CCCCSCCC(=O)OCCSCCOC</smiles>

It has to be emphasized that PTDG and PTTDG differ from PTG for the substitution of the methylene group in $\beta$-position respect to the ester group of the dicarboxylic acid subunit with an ether-oxygen or sulphur atom, respectively. In PTTTDG the ether-oxygen atom present in PTTDG is replaced by a sulphur, whereas PTDETDP differs from PPP for the substitution of the $-\mathrm{CH}_{2}$ - group in $\gamma$-position with respect to the ester group both of diol and dicarboxylic subunit with sulphur atoms. Lastly, PDEDG differs from PTTDG because of the presence of an ether-oxygen atom also in the glycol subunit.

At room temperature all the polyesters synthesized are semicrystalline solids, except PTTDG and PDEDG, which appear as viscous oils. The solubility of the polymers was checked in various solvents: all the samples showed a good solubility at room temperature in the most common organic solvents, i.e. chloroform, tetrachloroethane, methylene chloride, etc. 
Tab. 1. Molecular and thermal characterization data.

\begin{tabular}{cccccccccc}
\hline & \multicolumn{1}{c}{$1^{\text {st }}$ scan } & \multicolumn{4}{c}{$2^{\text {nd }}$ scan } \\
\hline Polymers & $M_{n}$ & $\begin{array}{c}T_{i d} \\
\left({ }^{\circ} \mathrm{C}\right)\end{array}$ & $\begin{array}{c}T_{\max } \\
\left({ }^{\circ} \mathrm{C}\right)\end{array}$ & $\begin{array}{c}T_{m} \\
\left({ }^{\circ} \mathrm{C}\right)\end{array}$ & $\begin{array}{c}\Delta H_{m} \\
(\mathrm{~J} / g)\end{array}$ & $\begin{array}{c}T_{g} \\
\left({ }^{\circ} \mathrm{C}\right)\end{array}$ & $\begin{array}{c}\Delta c_{p} \\
\left(\mathrm{~J} / g^{\circ} \mathrm{C}\right)\end{array}$ & $\begin{array}{c}T_{m} \\
\left({ }^{\circ} \mathrm{C}\right)\end{array}$ & $\begin{array}{c}\Delta H_{m} \\
(\mathrm{~J} / g)\end{array}$ \\
\hline PTG & 43500 & 377 & 398 & 54 & 60 & -49 & 0.600 & - & - \\
PPP & 49900 & 378 & 429 & 47 & 90 & -63 & 0.093 & 41 & 74 \\
PTDG & 15500 & 343 & 385 & 46 & 36 & -12 & 0.639 & - & - \\
PTTDG & 35700 & 354 & 391 & 64 & 49 & -21 & 0.520 & - & - \\
PDEDG & 20900 & 356 & 391 & - & - & -11 & 0.750 & - & - \\
PTTDG & 19300 & 330 & 369 & - & - & -37 & 0.600 & - & - \\
PTTTDG & 31700 & 346 & 373 & 36 & 3 & -45 & 0.520 & - & - \\
PTDETDP & 15400 & 334 & 359 & 34 & 2 & -45 & 0.650 & - & - \\
\hline
\end{tabular}
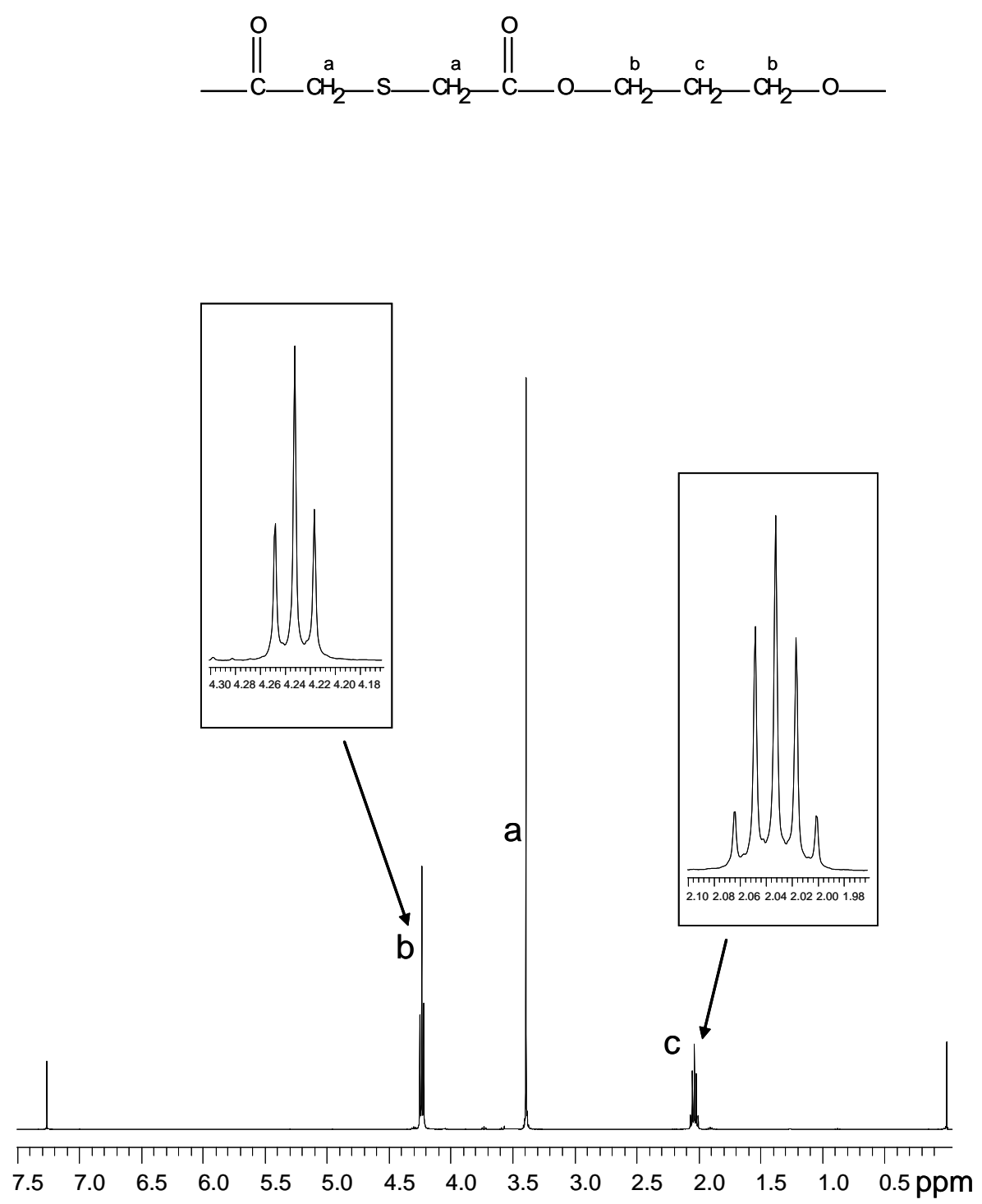

Fig. 1. $400 \mathrm{MHz}{ }^{1} \mathrm{H}-\mathrm{NMR}$ spectrum of PTTDG. 
Before molecular and thermal characterization, all the polymers were carefully purified by dissolution in chloroform and precipitation in cold methanol. Lastly, the samples were kept in a desiccator at room temperature for several days in order to remove the residual solvent. The synthesized polymers and their main characteristics are collected in Table 1.

First of all, it can be noted that all the polymers are characterized by relatively high molecular weights and this can be considered a proof that appropriate reaction conditions were obtained.

In order to have an understanding into the chemical structure, the ${ }^{1} \mathrm{H}-\mathrm{NMR}$ investigation on the samples was performed. As an example, the ${ }^{1} \mathrm{H}-\mathrm{NMR}$ spectrum of PTTDG is shown in Figure 1, together with the chemical shift assignments. In all cases the spectra were found to be consistent with the expected structure, indicating that no side reactions significantly occurred during the syntheses.

The polyesters were afterwards examined by thermogravimetric analysis and differential scanning calorimetry. The investigation on the thermal stability was carried out both in air and under nitrogen atmosphere. Figures 2 and 3 show the thermogravimetric curves of some samples in air.
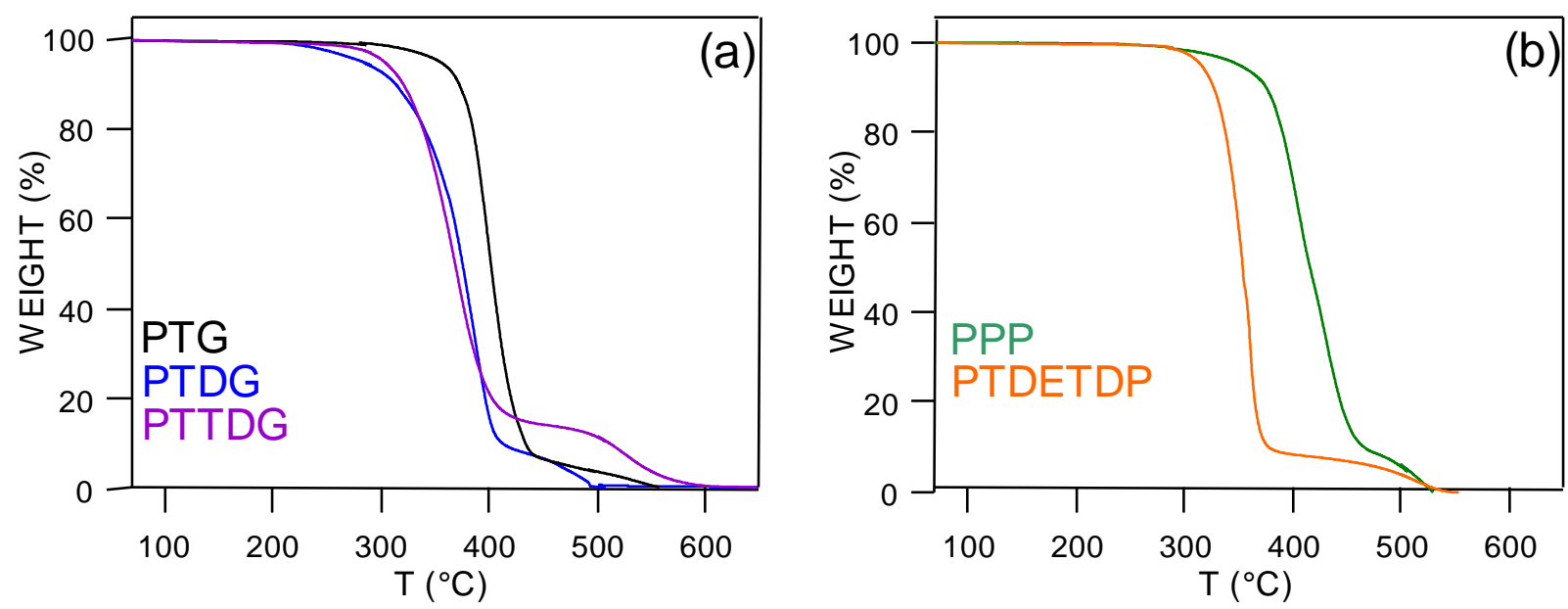

Fig. 2. Thermogravimetric curves of PTG, PTDG, PTTDG, PPP and PTDETDP in air (10 deg/min).

From the thermogravimetric curves in air, the temperature of initial decomposition $\left(T_{i d}\right)$ and the temperature corresponding to the maximum weight loss rate $\left(T_{\max }\right)$ were determined and collected in Table 1. As can be seen from Figure 2 and 3, in all cases the weight loss takes place practically in one-step and is $100 \%$. Moreover, as evidenced also by the temperature of initial decomposition ( $\left.T_{i d}\right)$ reported in Tab. 1, all the samples are characterized by a relatively high thermal stability, the $T_{i d}$ values ranging from 330 to $388^{\circ} \mathrm{C}$.

Anyway, a significant effect of the chemical structure of the polymer on its thermal stability can be observed. As is well known, at lower temperatures, linear aliphatic polyesters undergo depolymerization to linear and cyclic esters or lactones, a reaction commonly employed in the syntheses of many of such substances; at higher temperatures, a further decomposition ensues, typically with the formation of vinyl and carboxyl groups by ester scission and by cyclic elimination mechanism; a fourcentre cleavage to ketene groups has also been proposed [6]. Moreover, as reported 
in the literature, the main thermal degradation pathway of polyesters is highly dependent on the chain length of the diol subunit, whereas the influence of the dicarboxylic acid one is less important [7].
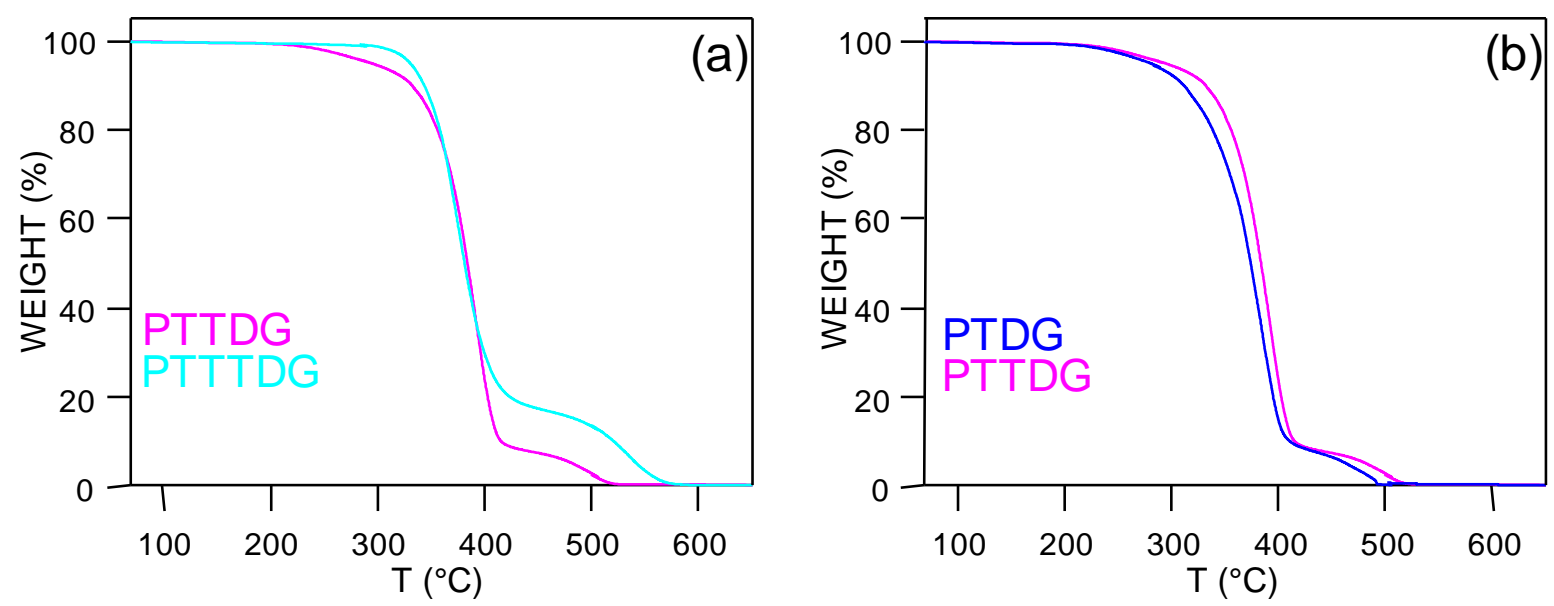

Fig. 3. Thermogravimetric curves of PTTDG, PTTTDG, PTDG and PTTDG in air (10 $\mathrm{deg} / \mathrm{min})$.

In our case, the different thermal stability of the polyesters synthesized cannot be explained exclusively on this basis, as the diol subunit is the same in some of the polymers investigated. Therefore, in some cases the observed behaviours have to be ascribed to different mechanisms involving the decomposition of the dicarboxylic acid subunits. As it can be seen from the Figure 2(a) and from the data collected in Table 1, PTG appears to be more thermally stable than PTDG, which is characterized by a slightly higher thermal stability than PTTDG. In fact, as documented in the literature [8], the introduction of ethero atoms along the polymer chains reduces the thermal stability, in particular favouring the thermooxidative processes of decomposition. Taking into account that among the various degradation mechanisms proposed to explain thermal degradation reactions occurring in polyesters there is also the random cleavage of covalent bonds of the polymeric chains, the higher stability of PTDG with respect of PTTDG can be explained on the basis of the higher energy of the C-O bond with respect to C-S one. In Figure 2(b), the thermogravimetric curves of PPP and PTDETDP in air are collected: PPP appears to be significantly more stable than PTDETDP. Again, the random fragmentation processes are more probable if weaker bonds are present. Therefore, the lower thermal stability of PTDETDP with respect to PPP can be ascribed to the C-S bond energy which is lower than $\mathrm{C}-\mathrm{C}$ ones.

In Figure 3(a) the effect of substitution of an ether-oxygen atom with a sulphur one is considered: again, the poly(thioether ester) is less stable than the corresponding poly(ether-oxygen ester), confirming the trend discussed above. Lastly, in Figure 3(b) the thermogravimetric curves of PTDG and PTTDG are reported: from their comparison, the effect of chain length of the diol subunit on the thermal stability can be evaluated. As it can be seen, PTTDG turned out to be more thermally stable than PTDG. The simplest way to interpret this result is to consider that in many cases aliphatic polyesters can be assimilated to polyethylene chains containing ester groups. In this view, it is well established that the introduction of ester linkages into the polyethylene chain reduces its thermal stability, the effect being proportional to 
their concentration [9]. Therefore, it is not surprising that PTTDG, which has a longer diol subunits, is characterized by a higher thermal stability with respect to PTDG. Moreover, it has to be taken into account that the most important mechanism in the decomposition of the diol subunit is the $\beta$-scission, shown both for PTDG and PTTDG in the schemes reported below.
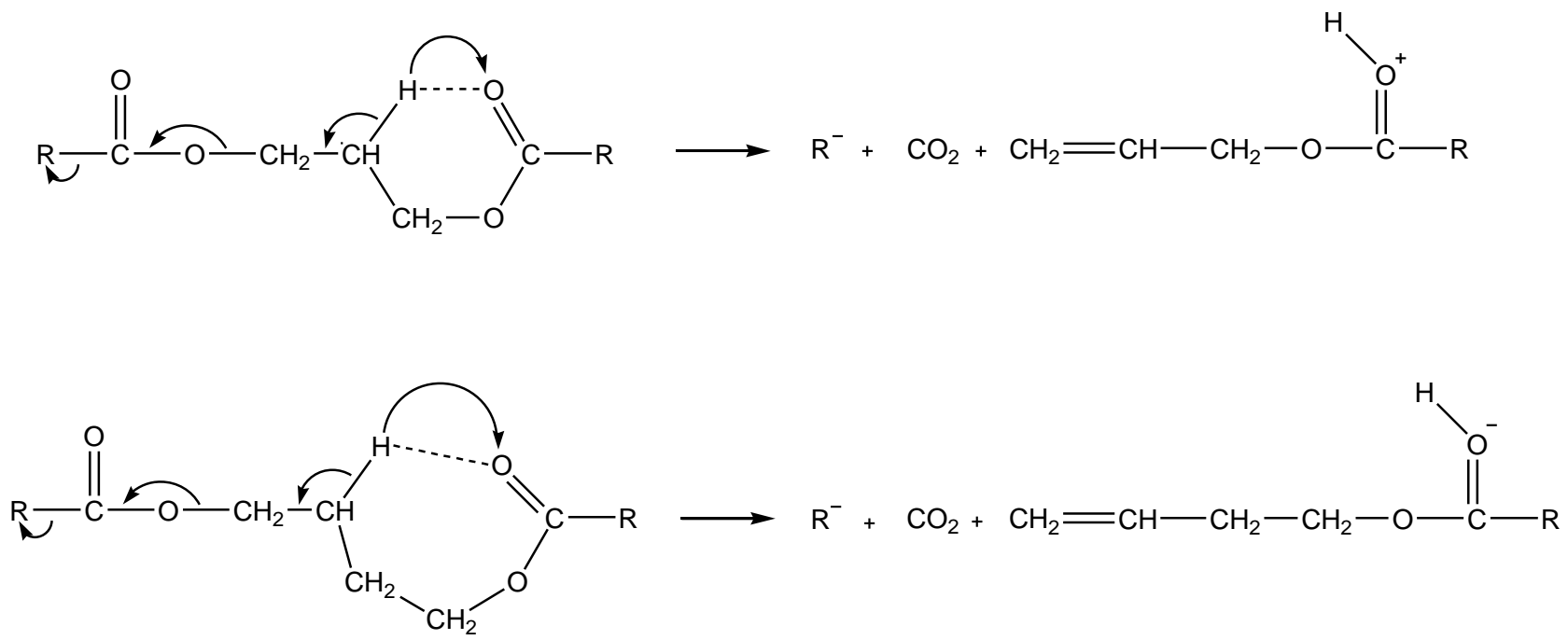

The lower thermal stability of PTDG can be also explained as due to the formation of a favorable six-membered transition state, as shown in the scheme. As a matter of fact, in the case of PTTDG a less stable seven membered structure has to be hypothesized.

Similar trends were obtained by means of TGA measurements carried out under nitrogen atmosphere. Therefore, it can be concluded that the introduction of ethero atoms ( $\mathrm{S}$ or $\mathrm{O}$ ) along the polymeric chain of an aliphatic polyesters favors the fragmentation processes and decreases the thermal stability. The effect appears to be more pronounced in the case of sulphur atoms.

As regard to calorimetric results, the samples being characterized are sufficiently high $M_{n} \mathrm{~S}$, an influence of molecular weight on the glass transition and melting phenomena of the polymers synthesized can be excluded.

It is well established that the melting behavior of a polymer is affected by its previous thermal history and therefore, in order to provide the same heat treatment to all the samples investigated, prior to thermal analysis the specimens have been aged for a very long time (about 6 months) at room temperature in desiccator. The DSC data obtained are collected in Table 1. First of all, it can be noted that the introduction of ethero atoms causes significant changes of $T_{g}$ and $T_{m}$ with respect to the corresponding poly(alkylene dicarboxylate)s. In order to evaluate the effect of introduction along the polymeric chain of the ethero atoms, appropriate comparisons can be made. In Figure 4 the calorimetric curves of PTG, PTDG, PTTDG, PPP and PTDETDP are shown. As evidenced by the chemical structures reported above, PTDG and PTTDG differ from PTG for the replacement of the methylene group of the dicarboxylic subunit in $\beta$-position with respect to the ester group with an oxygen or sulphur atom, respectively. The DSC traces of PTG and PTDG are typical of semicrystalline material, being characterized by the presence of a conspicuous melting endotherm. On the contrary, PTTDG appears as a completely amorphous polymer, as evidenced by the corresponding calorimetric curve, which shows only an endothermal baseline deviation associated to the glass transition. As far as the 
melting phenomenon is concerned, it can be noted that the melting point of the semicrystalline samples is affected by their chemical structure and appears to be lower for PTDG with respect to PTG. This result is in good agreement with those previously obtained, in investigating poly(butylene naphthalate) and poly(diethylene naphthalate) [10] and can be explained on the basis of a lower symmetry of the chains in the ether-linkage containing polyesters. Moreover, the lower heat of fusion of PTDG with respect to PTG indicates that this latter is characterized by a higher capability of crystallizing. Also this trend can be explained as due to the higher symmetry of the chains of PTG. In the case of PTTDG the ability to crystallize is completely undone: as a matter of fact, in this case, the intermolecular interactions, which stabilize the alignment of the macromolecules and therefore favor crystallization, are weaker on respect to PTDG (oxygen atoms are indeed characterized by higher electronegativity than sulphur ones). Figure 4 compares also the first DSC scans of PPP and PTDETDP. As shown by the chemical structure reported above, in PTDETDP two methylene groups, one belonging to diol subunit and the other to dicarboxylic one, are replaced by sulphur atoms. First of all, it can be noted that PTDETDP is much less crystalline than PPP, as evidenced by its significantly lower heat of fusion. Also, the $T_{m}$ of PTDETDP is consistently lower than that of PPP, in agreement with results previously obtained [9]; again these results can be ascribed to the lower symmetry of the chains in the thioether-linkage containing polyester.
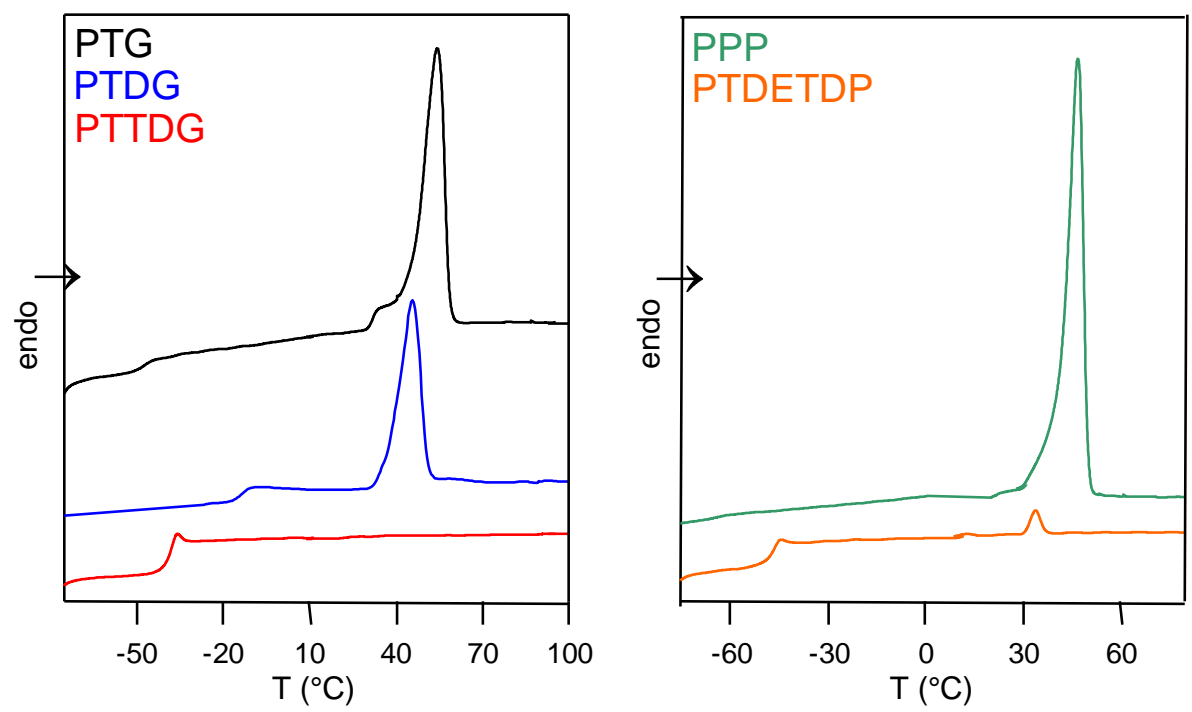

Fig. 4. Calorimetric curves ( $1^{\text {st }}$ scan) of PTG, PTDG, PTTDG, PPP and PTDETDP at heating rate of $20 \mathrm{deg} / \mathrm{min}$.

A confirmation of the trend of the melting point discussed above can be obtained comparing the first scans of PTTDG and PDEDG (see Figure 5). This latter differs from PTTDG because of the introduction of ether-oxygen atom in the diol subunit. The calorimetric trace of PTTDG is typical of a semicrystalline material, a glass transition and a conspicuous melting endotherm being evident. The phase behaviour of PDEDG is opposite: as matter of fact, PDEDG is completely amorphous, the corresponding DSC curve being characterized only by an endothermal baseline deviation. Again the introduction of an etheroatom along the polymeric chain causes a reduction of the symmetry as well as of the mobility (see in the following), completely undoing the capacity of crystallizing. A different consideration can be 
made comparing the DSC traces of PTDG and PTTDG which contain trimethylene and tetramethylene diol subunit, respectively. As it can be seen from Figure 5, PTTDG melts at higher temperature with respect to PTDG, showing the well known odd-even effect on the basis of which the polyesters with an even number of carbon atoms per repeat unit have higher melting points than those characterized by an odd number of carbon atoms. Therefore, in addition to the chain flexibility, the characteristics of the chain conformation and the crystal structure have to be also considered to explain the observed behaviour.

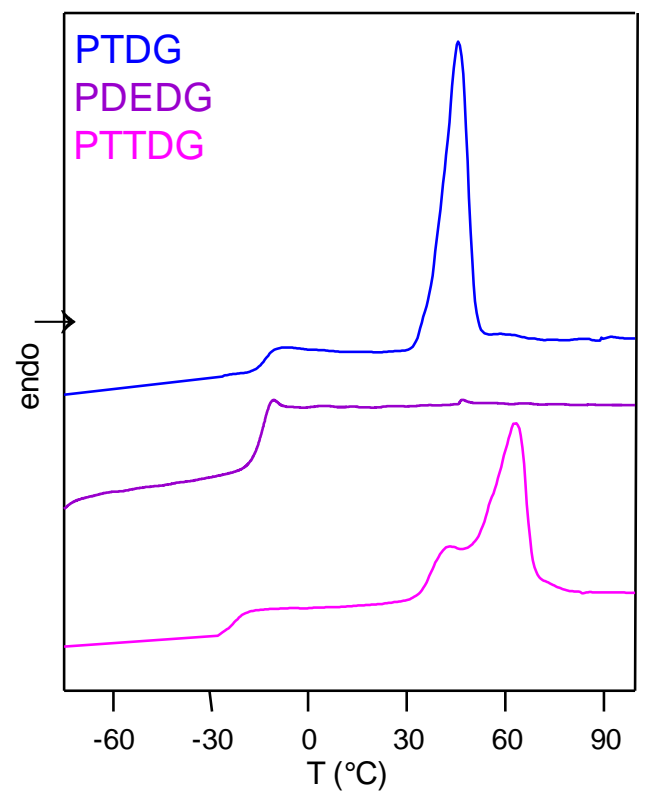

Fig. 5. Calorimetric curves ( $1^{\text {st }}$ scan) of PTDG, PDEDG and PTTDG at heating rate of $20 \mathrm{deg} / \mathrm{min}$.

As is well known, a partially crystalline material usually exhibits different glass transition behaviour than the completely amorphous sample. In fact, although some conflicting results are reported in the literature [11], crystallinity usually acts like cross-linking and raises $T_{g}$ through its restrictive effect on the segmental motion of amorphous polymer chains. Therefore, in order to study the influence of the chemical structure on the glass transition of a polymer, the phenomenon should be examined in the total absence of crystallinity. In this view, all the samples under investigation were subjected to rapid cooling (quenching) from the melt (see the Experimental section for the details). The data of the second DSC scans are collected in Table 1: as it can be seen, all the samples under investigation, after melt quenching are completely amorphous, with the exception of PPP, whose calorimetric curve is characterized by an endothermal baseline deviation associated with the glass transition and by a conspicuous melting endotherm. This is due to the fact that the crystallization rate of the polymer is higher than the cooling one. By comparing the $T_{g}$ values measured, one can remark as follows:

(i) the substitution of a methylene group with an ethero atom induces a significant increment of the glass transition temperature, independent of the kind of atom introduced (see PTG vs. PTDG, PPP vs. PTETDP, PTG vs. PTTDG, PTTDG vs. PDEDG). To explain this trend it has to be taken into account that the presence along the polymer chains of ethero atoms, such as ether-oxygen and sulphur, can cause 
two effects: 1) the polymer chain becomes more flexible; 2) the polymer chain mobility decreases, because of the stronger interchain interactions. The latter are due to the higher electronegativity of $O$ and $S$ with respect to $C$ atoms, which give rise to the formation of $\mathrm{C}-\mathrm{O}$ and $\mathrm{C}-\mathrm{S}$ polar bonds. As reported in the literature [9], usually the first effect prevails when ethero atoms are introduced along the polymeric chain of aromatic polyesters. The second one is, on the contrary, predominant in the case of aliphatic ones [12-13]. The results discussed in the present paper confirm this trend.

(ii) The replacement of ether-oxygen atoms with sulphur ones gives rise to a lowering of the glass transition temperature, (see PTDG vs. PTTDG, PTTDG vs. PTTTDG). This result can be explained on the basis of two effects: 1) the larger dimension of sulphur atoms with respect to the oxygen ones, which therefore are able to form S-C bonds longer than the $\mathrm{O}-\mathrm{C}$ ones, makes the polymer chains more flexible; 2) the lower electronegativity of sulphur atoms with respect to the oxygen ones, which gives rise to less polar C-S bonds and therefore to weaker interchain interactions. It is worth emphasizing that a similar behaviour has been already observed in other sulphur-containing polymers [10, 14-17].

(iii) The glass transition temperature steadily decreases as the number of $-\left(\mathrm{CH}_{2}\right)$ groups per repeat unit is increased (see PTG vs. PPP, PTDG vs. PTTDG, PTTDG vs. PTTTDG). This trend can be explained on the basis of an increment of the chain flexibility: as a matter of fact, the higher is the number of methylene groups in the polymeric chain the lower the concentration of the stiffer ester groups (O-CO-). As a consequence, the polymeric chain is more flexible and thus the polymer has a lower $T_{g}$.

\section{Conclusions}

The data obtained on the polyesters synthesized display that small modifications of the chemical structure, such as the substitution of a methylene group with an ethero atom (oxygen or sulphur), can lead to significant variations in the thermal properties, whose entity depends on the kind of ethero atoms introduced. In particular:

- the thermal stability slightly decreases, the effect being more pronounced in the case of sulphur atoms;

- the glass transition temperature increases, due to interchain interactions because of the electronegativity of the atoms; in this case the variation is larger in the case of ether-oxygen atoms;

- the melting point decreases, due to the lower symmetry of the chain;

- the ability to crystallize decreases, due to a reduction of the symmetry as well as of the mobility of the chain.

\section{Experimental part}

\section{Reagents}

Pimelic acid (PA), dimethylglutarate (DMG), diglycolic acid (DG), thiodiglycolic acid (TDG), dimethylthiodipropionate (TDP), 1,3-propanediol (TD), 1,4-butanediol (TTD), 1,5-pentanediol (PP), diethylene glycol (DEG), thiodiethylene glycol (TDEG) and titanium tetrabutoxide $\left(\mathrm{Ti}(\mathrm{OBu})_{4}\right)$ (Aldrich) were reagent grade products; all the 
reagents were used as supplied, except $\mathrm{DEG}$ and $\mathrm{Ti}(\mathrm{OBu})_{4}$, which were distilled before use.

\section{Synthesis of polymer samples}

Poly(pentamethylene pimelate) (PPP) and poly(trimethylene glutarate) (PTG), were synthesized in bulk starting from PA or DMG and PP or TD, using a $100 \mathrm{~mol} \%$ excess of the glycol with respect of carboxylic acid; poly(trimethylene diglycolate) (PTDG), poly(tetramethylene diglycolate) (PTTDG), poly(trimethylene thiodiglycolate) (PTTDG), poly(tetramethylene thiodiglycolate) (PTTTDG), poly(diethylene diglycolate) (PDEDG) and poly(thiodiethylene thiodiglycolate) (PTDETDP) were also obtained by melt polycondensation procedure from diglycolic acid, or thiodiglycolic acid or dimethylthiodipropionate, and 1,3-propanediol or 1,4-butanediol, diethylene glycol or thiodiethylene glycol, according to the kind of polyester to be prepared (100 mol \% excess of the glycol with respect to carboxylic acid). In all cases $\mathrm{Ti}(\mathrm{OBu})_{4}$ was employed as catalyst (about $0.2 \mathrm{~g}$ of $\mathrm{Ti}(\mathrm{OBu})_{4} / \mathrm{kg}$ of polymer). The syntheses were carried out in a $200 \mathrm{~mL}$ stirred glass reactor, with a thermostatted silicon oil bath; temperature and torque were continuously recorded during the polymerization. The polymers were obtained according to the usual two-stage polymerization procedure. In the first stage, under pure nitrogen flow, the temperature was increased up to 180 ${ }^{\circ} \mathrm{C}$ and maintained there for until more than $90 \%$ of the theoretical amount of methanol or water was distilled off (typically, about 2 hours). In the second stage the pressure was reduced, in order to facilitate the removal of the glycol in excess and the temperature was kept at $200{ }^{\circ} \mathrm{C}$ until a torque constant value was measured. The polymers obtained, because of the use of $\mathrm{Ti}(\mathrm{OBu})_{4}$ as catalyst and the high temperature, which favor the redistribution reactions, are statistical in molecular weight distribution.

\section{${ }^{1} H$-NMR spectroscopy}

The chain structure of the polymers were determined by means of ${ }^{1} \mathrm{H}-\mathrm{NMR}$ spectroscopy. Polymer samples were dissolved $(10 \mathrm{mg} / \mathrm{mL})$ in chloroform-d with $0.03 \%(\mathrm{v} / \mathrm{v})$ tetramethylsilane added as an internal standard. The measurements were carried out at room temperature, employing a Varian XL-400 instrument.

\section{Gel-permeation chromatography}

Molecular weight data were obtained by gel-permeation chromatography at $30{ }^{\circ} \mathrm{C}$ using a 1100 Hewlett Packard system equipped with PL gel $5 \mu$ MiniMIX-C column (250/4.6 length/i.d., in mm). A refractive index was employed as detector. In all cases, chloroform was used as eluent with a $0.3 \mathrm{ml} / \mathrm{min}$ flow, and sample concentrations of about $2 \mathrm{mg} / \mathrm{mL}$ were applied. A molecular weight calibration curve was obtained with several polystyrene standards in the range of molecular weight 2,000-100,000.

\section{Thermal analysis}

\section{-TG measurements}

Thermogravimetric analysis was carried out both in air and under nitrogen atmosphere using a Perkin Elmer TGA7 apparatus (gas flow: $40 \mathrm{~mL} / \mathrm{min}$ ) at 10 ${ }^{\circ} \mathrm{C} /$ min heating rate up to $900{ }^{\circ} \mathrm{C}$. The procedure suggested by the supplier was 
followed for the temperature calibration of equipment. This method is based on the change of the magnetic properties of two metal samples (Nickel and Perkalloy) at their Curie points $\left(354.0\right.$ and $596.0^{\circ} \mathrm{C}$, respectively). The $T_{\text {onset }}$ at which the weight loss starts was taken as the temperature of initial decomposition $\left(T_{i d}\right)$.

\section{DSC measurements}

Calorimetric measurements were carried out by means of a Perkin Elmer DSC7 instrument equipped with a liquid sub ambient accessory and calibrated with high purity standards (indium and cyclohexane). With the aim of measuring the glass transition and the melting temperatures of the polymers under investigation, the external block temperature control was set at $-120{ }^{\circ} \mathrm{C}$ and weighed samples of c.a. $10 \mathrm{mg}$ were encapsulated in aluminum pans and heated to about $40{ }^{\circ} \mathrm{C}$ above fusion temperature at a rate of $20^{\circ} \mathrm{C} / \mathrm{min}$ (first scan), held there for $3 \mathrm{~min}$, and then rapidly quenched (about $100{ }^{\circ} \mathrm{C} / \mathrm{min}$ ) to $-80^{\circ} \mathrm{C}$. Finally, they were reheated from $-80{ }^{\circ} \mathrm{C}$ to a temperature well above the fusion temperature of the sample at a heating rate of 20 ${ }^{\circ} \mathrm{C} / \mathrm{min}$ (second scan). The glass-transition temperature $T_{g}$ was taken as the midpoint of the heat capacity increment $\Delta c_{p}$ associated with the glass-to-rubber transition. The melting temperature $\left(T_{m}\right)$ was determined as the peak value of the endothermal phenomenon in the DSC curve. The specific heat increment $\Delta c_{p}$, associated with the glass transition of the amorphous phase, was calculated from the vertical distance between the two extrapolated baselines at the glass transition temperature. The heat of fusion $\left(\Delta H_{m}\right)$ of the crystal phase was calculated from the area of the DSC endotherm.

\section{References}

[1] Gross, R.A.; Kumar, A; Karla, B Chem. Rev. 2001, $101(7), 2097$.

[2] Kobayashi, S; Uyama, H; Kimura, S; Chem. Rev. 2001, 101(12), 3793.

[3] Gross, R.A.; Karla, B; Kumar, A Appl. Microbiol. Biotechnol. 2001, 55(6), 655.

[4] Lotti, N.; Finelli, L.; Fiorini, M.; Righetti, M.C.; Munari, A. Polymer 2000, 41, 5297.

[5] Lotti, N.; Finelli, L.; Fiorini, M.; Righetti, M.C.; Munari, A. J. Appl. Polym. Sci. 2001, $81,981$.

[6] Goodman, I. Encyclopaedia of Polymer Science and Engeneering, 2nd ed. vol.12, New York: Wiley, 1988, pp. 1-75.

[7] Plage, B.; Schulten, H.R. Macromolecules 1990, 23(10), 2642.

[8] Zimmermann, H. Degradation and Stabilization of Polyesters, Applied Science Publishers, 1984, (chapter 3).

[9] Korshak, V.V. and Vinogradova, S.V. Polyesters Pergamon Press: Oxford, 1965, (chapter. 6).

[10] Soccio, M.; Finelli, L.; Lotti, N.; Siracusa, V.; Ezquerra, T.A.; Munari, A. J. Polym. Sci. Part B: Polym. Phys. 2007, 45, 1694.

[11] Boyer, RF. Rub. Chem. Tech. 1963, 36, 1303.

[12] Cao, A.; Okamura, T.; Nakayama, K.; Inoue, Y.; Masuda, T. Polym. Degrad. Stab. 2002, 78(1), 107.

[13] Soccio, M.; Lotti, N.; Finelli, L.; Gazzano, M.; Munari, A. Eur. Polym. J. 2008, 44(6), 1722.

[14] Berti, C.; Celli, A.; Marianucci, E. Eur. Polym. J. 2002, 38, 1281.

[15] Berti, C.; Celli, A.; Marianucci, E.; Vannini, M. Eur. Polym. J. 2005, 41, 1812.

[16] Lotti, N.; Finelli, L.; Messori, M.; Munari, A. e-Polymers 2006, nº 017. 
[17] Lotti, N.; Siracusa, V.; Finelli, L.; Marchese, P.; Munari, A. Eur. Polym. J. 2006, 42, 3374. 